\title{
Supermirrors study by neutron multireflection
}

\author{
B. BALLOT, A. MENELLE, K. AL USTA* , B. FARNOUX and F. SAMUEL* \\ Laboratoire Léon Brillouin, CEA-CNRS, CE Saclay, 91191 Gif-sur-Yvette cedex, France \\ ${ }^{*}$ Cilas, Route de Nozay, BP. 27, 91460 Marcoussis, France
}

\begin{abstract}
We present here time of flight neutron multireflection measurements. This technique has been developed at the Laboratoire Léon Brillouin (Saclay-France). Used in the investigation of supermirrors $\mathrm{NiC} / \mathrm{Ti}$, it allows us to increase the precision of the measurement of the most important part of the curve for technological applications : the extension of the total reflection plateau. The results on three systems $\left({ }^{58} \mathrm{Ni}\right.$ single layer, multilayer $\mathrm{NiC} / \mathrm{Ti}$, supermirror $\mathrm{NiC} / \mathrm{Ti}$ ) are presented in this paper. Beyond the good quality of the mirrors, they demonstrate the very good reliability of such a measurement. Moreover, they show that compared to a classical single reflection measurement, multireflection experiment enables us to increase the accuracy of the reflectivity value measured on the extended plateau.
\end{abstract}

\section{INTRODUCTION}

Due to the recent development of the neutron scattering and reflectometry techniques, high quality neutron optics (metallic multilayers) are needed to improve the performances of neutron guides. Special kinds of multilayers are supermirrors. They are made of aperiodic layers of alternately deposited low index (reflectant) and high index (spacer) layers ${ }^{1}$. Compared to classical nickel coated mirrors, supermirrors allow to extend the total reflection plateau. This extension is created by a succession of Bragg peaks that correspond to the different thicknesses of the bilayers present in the aperiodic multilayers. Our systems are made with $\mathrm{NiC} / \mathrm{Ti}$ layers where nickel carbide is the reflectant material and titanium is the spacer. Supermirrors were used in the built of a new neutron guide at the LLB. They enable a spectacular increase of the transmitted flux ${ }^{2}$.

For technological applications, the most interesting part of the reflectivity curves is situated in regions where the reflectivity value is close to one, that is on the extended plateau, just before the apparent cut off of the mirror. Using a standard single reflection experiment, a very long counting time is required in order to obtain the statistical accuracy needed to measure variations of reflectivity less than $1 \%$. In order to improve the precision of the measurements on the extended plateau (by increasing the difference between the direct and reflected beam), we have installed a time of flight neutron multireflection experiment at the Laboratoire Léon Brillouin (LLB). In a multireflection experiment with $\mathrm{N}$ reflections the measured value is $\mathrm{R}^{\mathrm{N}}$ instead of $\mathrm{R}$ for a single reflection experiment. The precision of the measurement is all the more important since the number of reflections $\mathrm{N}$ is high. Moreover, the result of the measurement is due to all the surface of the mirror contrary to the single reflection measurement. 


\section{PRINCIPLES OF MULTIREFLECTIVITY}

In the case of a single reflection, the reflected intensity $I$ is proportional to the intensity of the direct beam $\mathrm{I}_{0}$ for a value of the momentum transfer $\mathrm{q}$. The proportional coefficient is the reflectivity value of the mirror $\mathrm{R}$.

$$
\begin{aligned}
& \mathrm{I}(\mathrm{q})=\mathrm{RI}_{0}(\mathrm{q}) \\
& \mathrm{q}=\frac{2 \pi \sin \theta}{\lambda}
\end{aligned}
$$

For a multireflection experiment and for a number $\mathrm{N}$ of reflections ( $\mathrm{N}$ is integer), the intensity measured is :

$$
I(q)=R^{N} I_{0}(q)
$$

so that the value of multireflectivity $R^{\prime}$ is defined as a function of the single reflectivity value $R$ on the same mirror:

$$
\mathbf{R}^{\prime}=\mathbf{R}^{\mathrm{N}}
$$

When the number of reflection is not an integer, that means when a part of the beam is reflected $\mathrm{N}$ times and the other part $N+1$ times (see figure 1), the measured reflectivity is :

$$
\mathbf{R}^{\prime}=(1-\mathrm{x}) \mathbf{R}^{N}+\mathrm{xR}^{\mathrm{N}+1}
$$

where $N$ and $x$ are functions of the length of the mirrors $L$, the incident angle $\theta$, and the width between the two mirrors e.

$$
L=(N+x) \frac{e}{\tan \theta}
$$

One can note here that the beam divergence and the surface roughness are not considered in the calculation of $\mathbf{R}^{\prime}$ in expression (5). This expression does not present an analytical solution and should be solved numerically. Experimentally, the angle $\theta$ is chosen so that the number of reflections is integer :

$$
\theta_{\mathrm{N}}=\arctan \left(\frac{\mathrm{eN}}{\mathrm{L}}\right)
$$

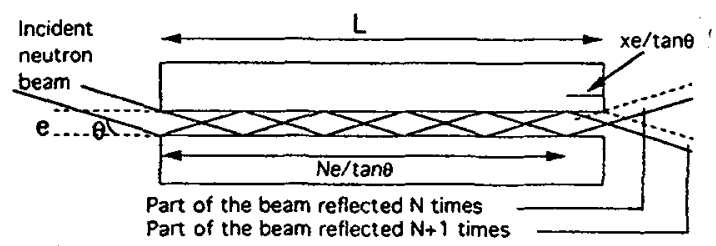

Figure 1 : Beam position for multireflection when the last reflection is not complete
(7)

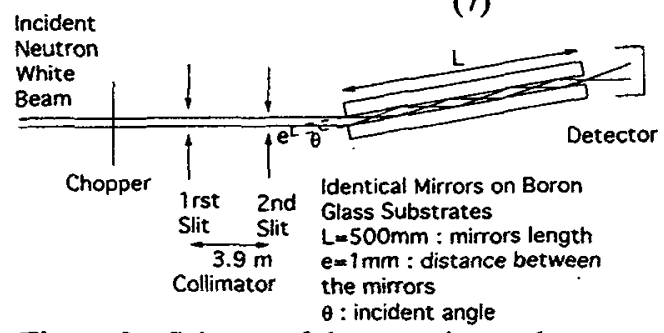

Figure 2 : Schema of the experimental setup

\section{EXPERIMENTAL SETUP}

The measurements have been done on the reflectometer EROS at the LLB (Saclay). It is a time of flight (TOF) neutron spectrometer. Compared with a monochromatic experiment, TOF allows to keep constant the angle $\theta$ and thus the number of reflections $N$ during a measurement. The wavelength range is $\lambda=3 \AA$ to $23 \AA$. The two identical mirrors surfaces are parallel and separated with three little metallic spacers of $1 \mathrm{~mm}$ thick put on the edge of the mirrors. The mirrors are deposited on boron glass substrates that allows us to use the mirrors as slits. This set up allows a very good measurement reproducibility. Two measurements with $\theta$ and $-\theta$ are made. Mirrors are inclined with the angles $\theta$ and 
$\theta$ chosen in order to obtain an entire number of reflections $N$. The incident beam is measured for $\theta=0$. The experimental setup is described on figure 2 . The beam intensity is measured as a function of $\lambda$.

\section{SYSTEMS STUDIED}

Three different pairs of mirrors have been measured by classical one reflection and multireflection methods. They are the following ones : $i$ )mirrors of a $1200 \AA{ }^{58} \mathrm{Ni}$ single layer, $\left.i i\right)$ multilayers $(\mathrm{NiC} / \mathrm{Ti})$, iii)supermirrors (NiC/Ti).

The multilayers are made of series of bilayers NiC/Ti. The total reflection plateau (that ends in $\mathrm{Q}_{\mathrm{c}}$ ) is due to a thick Ni layer deposited on the top of the stack. The bilayers periodicity has been calculated so that the corresponding Bragg peak is situated just after $Q_{c}$. It creates thus an extension of the total reflection plateau.

Various angles from $\theta_{\mathrm{N}}=0.57$ to 1.37 degrees have been used for multireflection measurements so that the number of reflections $\mathrm{N}$ varies from 5 to 12 .

\section{V.RESULTS}

The multireflection results described here have been obtained for a number of reflections $\mathrm{N}=7$. Figure 3 displays the experimental reflectivity curve $R(q)$ of the classical single reflection measurement. The seventh root $\left(\sqrt[7]{R^{\prime}}\right)$ of the measured reflectivity $R^{\prime}$ resulting from multireflection is also plotted in figure 3 as a function of $q$. These two curves have been obtained with the ${ }^{58} \mathrm{Ni}$ single layer sample. The reflectivity value on the total reflection plateau is larger than 0.98 in the two cases. This value of the reflectivity shows the very good quality of the ${ }^{58} \mathrm{Ni}$ mirror. The perfect agreement between the two curves confirm the interest of the multireflection measurement. One can see in figure 3 that, using a similar counting time, the experimental error is decreased by the use of multireflection. The precision of the reflectivity measurement in the region of the plateau is thus markedly increased.

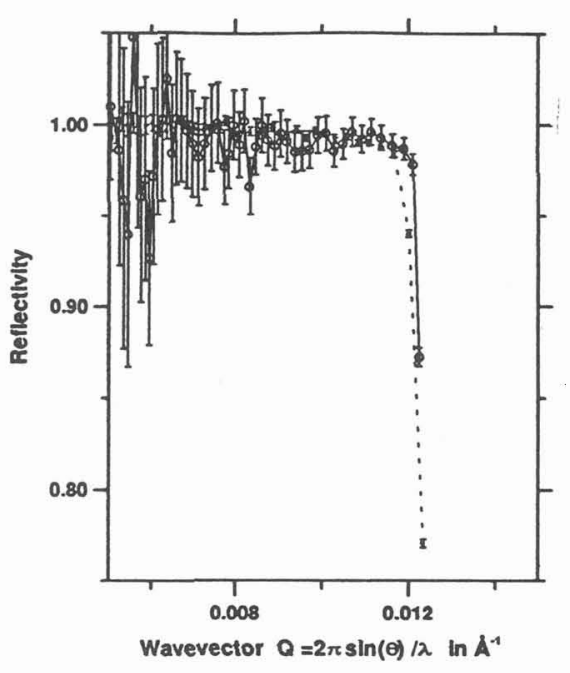

Figure 3 : Reflectivity measurement $\mathbf{R}$ by classical single reflection (solid line) and calculated $\sqrt[7]{R^{\prime}}$ (dotted line) from multireflection measurement $R^{\prime}$ for 7 reflections on ${ }^{58} \mathrm{Ni}$ mirror.

In addition to a better precision in the measurement of reflectivity on the plateau, multireflection experiments emphasise variations of reflectivity. It enables thus to visualise small features of the curve. As an example, figure 4 presents the reflectivity measurements obtained on the multilayers by the classical single reflection $R$ and by multireflection $R^{\prime}$. On $R^{\prime}$, the reflectivity value is 0.844 on the extended plateau. This value is $15.6 \%$ lower than 1 . On $R$, this value is only $2.4 \%$. Multireflection measurements allow thus to see clearly the important step between the total reflection plateau and the extended one. This step on $R^{\prime}(q)$ allows to visualise clearly the two distinguished phenomena that are responsible of the plateau : the first part of the plateau is due to the reflectant material NiC while the 
second is characteristic of the multilayers stack. Moreover, the shape of the Bragg peak is strongly marked on the $R^{\prime}$ curve. This is not true for $R$. Multireflection experiment emphasises the characteristic answer of the mirror. The interest is thus to determine unambiguously the performances of the mirrors.

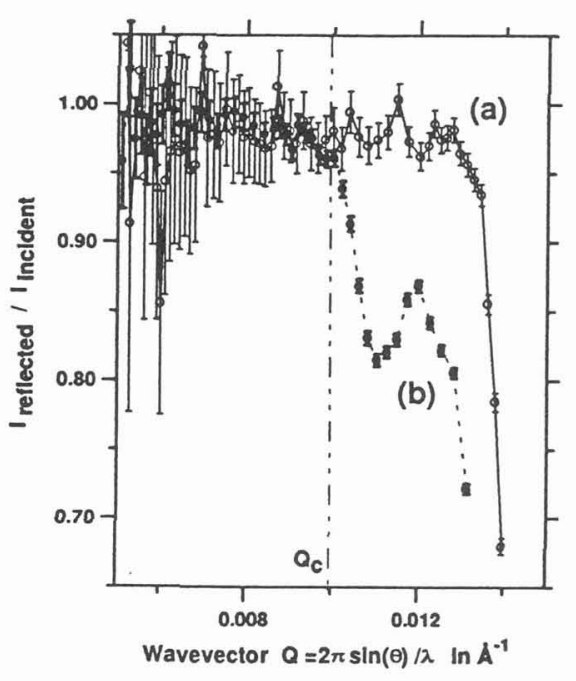

Figure 4 : Reflected intensity on incident intensity measured on multilayers $\mathrm{NiC} / \mathrm{Ti}$ with $a$ ) classical single reflection $(\mathrm{R}), b) 7$ reflections ( $\left.\mathrm{R}^{\prime}\right)$.

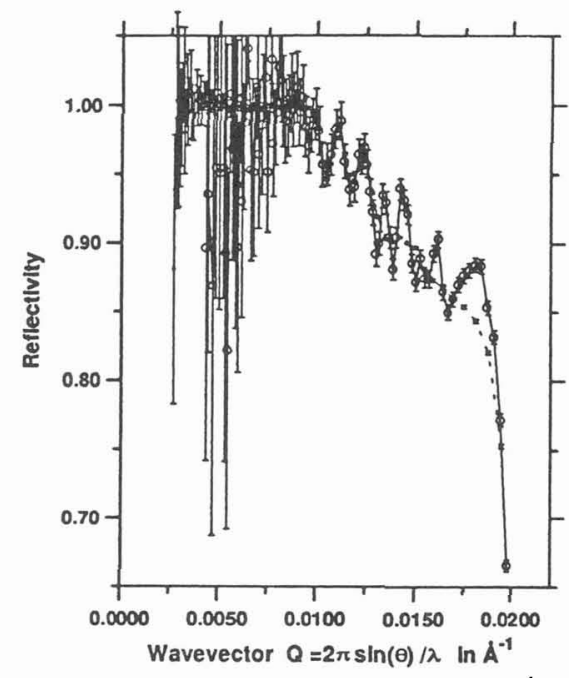

Figure 5 : Reflectivity measurement $R$ by classical single reflection (solid line) and calculated $\sqrt[3]{\mathrm{R}^{\prime}}$ (dotted line) from multireflection measurement $\mathrm{R}^{\prime}$ for 7 reflections on supermirrors.

The experimental results on supermirror are shown on figure 5 . The reflectivities $R$ and $\sqrt[2]{R^{\prime}}$ are plotted. $R$ and $R^{\prime}$ were measured by classical single reflection and multireflection respectively. $\sqrt[7]{R^{\prime}}$ has been calculated for each $\mathbf{q}$ from the measurement $R^{\prime}$. The fact that the two curves superimpose proves once again that the relation $R=\sqrt[7]{R^{\prime}}$ is confirmed experimentally. The important decrease of the experimental error allows a much better precision on the measurement of the reflectivity value on the supermirror extended plateau.

\section{CONCLUSION}

We have developed a time of flight neutron multireflection measurement method at the Laboratoire Léon Brillouin (Saclay-France). The measurements have been made on three different systems : ${ }^{58} \mathrm{Ni}$ single layer, multilayer and supermirror. Compared to a classical single reflection measurement, they show the important increase of the precision on the extended plateau reflectivity value obtained by multireflection. This measurement method allows us to simulate what really occurs in a guide such as those installed at the Orphée reactor.

\section{REFERENCES}

1. J.B. HAYTER, H.A. MOOK, J. Appl. Cryst., 22, 35-41, 1989

2. B. BALLOT, F. SAMUEL, B. FARNOUX, SPIE Proc., 1738, 159-165, San Diego, 1992 